In the largest study of its kind, diva lp roex sodium (Depa kote ${ }^{\circledR}$ ) a nd lithium are shown to be safe and efficacious in reducing symptoms of mania in children with bipolar 1 disorder.

\title{
Controlled trial of widely used mood stabilizers demonstrates efficacy in pediatric bipolar disorder
}

$N$ ew results from the largest pediatric study of its kind have explored the effects of divalproex sodium

(D epakote ${ }^{\circledR}$ ) in a moodstabilizing trial for children and adolescents with bipolar disorders. The drug has been shown to be effective in significantly improving the symptoms of bipolar disorder.

D rugs for the treatment of bipolar disorder in children and adolescents are few in number as many have not been indicated by the US FD A as being suitable for use in younger patients; research has focused on dosing safety and efficacy in the pediatric patient.

The study has offered confirmation of the efficacy of current medication for pediatric bipolar disorder: dival proex and lithium.

"O ur study now proves that these agents definitely work, which may give clinicians and families peace of mind", explained Robert Kowatch, a child and adolescent psychiatrist specializing in bipolar and other mood disorders at Cincinnati Childrens' H ospital, OH, USA, the main center for the recent study.

The Pediatric Bipolar Collaborative M ood Stabilizer Trial, is the first controlled trail to investigate and compare lithium and divalproex versus placebo for 6 months. This is the largest study to date involving lithium and divalproex and the first to demonstrate efficacy in children aged 7 to 17 years with bipolar 1 disorder, mixed or manic.

Investigators randomly assigned 153 children to receive a placebo, lithium or dival proex. The trail was divided into stages; neither the investigators nor patients knew to which treatment group the patients were assigned at the end of the 8-week treatment phase. Those patients who responded to treatment could continue in the double-blind testing for a further 16 weeks.

After the first 8-week phase, patients receiving dival proex showed a significant decrease in manic symptom severity, using the Young M ania Rating Scale (YM RS) as the primary outcome measure.

In total, $56 \%$ of patients in the dival proex group, $41 \%$ in the lithium-treated group and $30 \%$ of patients in the placebo group experienced more than a 50\% decrease in YM RS scores.

Other primary outcome measurements included the Clinical Global Impressions Ratings (CGI) improvement score. The study indicated that $54 \%$ of the dival proex group, $42 \%$ of the lithium group and $29 \%$ of the placebo group improved their CGI scores by 1 or 2 points.

Bipolar disorder also known as bipolar 1 disorder, effects approximately $1 \%$ of children aged 19 years and younger, usually occurring in children aged between 6 and 17 years. M ain symptoms include severe, explosive mood swings, euphoria, irritable mood and decreased need for sleep without daytime fatigue, pressured speech that is difficult to interrupt, racing thoughts and distractibility that varies with mood, increased goaldirected activity. Increased rates of suicide are also attributed to the disease, with most children being affected for life and requiring life-long medication regimes, psychotherapy and environmental adjustments, such as stress reduction, strict sleep regimes and avoidance of stimulant drugs including caffeine and alcohol.

The study was supported by The N ational Institute of $M$ ental $\mathrm{H}$ ealth.

Source: Pfeifer JC, Kowatch RA, DelBello MP: The use of antipsychotics in children and adolescents with bipolar disorders. Expert 0 pin. Pharmacother. 8(16), 2673-2687 (2007)

\section{O rgan- rejection drug} increases risk of birth defects and miscarriage

The U S FD A has warned that the drug C ellC ept ${ }^{\circledR}$, used to prevent organ rejection after transplantation, carries an increased risk of birth defects and miscarriages.

Cellcept is used to prevent organ rejection among kidney, liver and heart transplant recipients, but increases the likelihood of birth defects and miscarriage during the first trimester of pregnancy. Facial and ear deformities, limb problems and issues with the heart and other organs have all been linked to the use of the drug during pregnancy.

Postmarketing data released by Roche, the company involved, showed that out of 77 women who took C ellC ept, there were 25 miscarriages and 14 defects among fetuses and infants. Six of the defects were ear abnormalities.

TheFD A has stated that women of childbearing age should have a negative pregnancy test within 1 week before beginning treatment with CellC ept, and receive birthcontrol counseling and use effective contraception to avoid pregnancy during treatment. Source: Press release form Roche: www.rocheusa.com/products/cellcept /pregnancy_notice.pdf FD A report on CellC ept: www.fda.gov/medwatch/safety/200 7/C ellC ept_PI.pdf - 


\section{in brief...}

Children with autism: effect of iron supplementation on sleep and ferritin. Dosman CF, Brian JA, Drmic IE, Senthilselvan A et al.: Pediatr. Neurol. 36(3), 152-158 (2007). Determines the relationship between low serum ferritin and sleep disturbance in children with autism spectrum disorder. An 8-week openlabeled trial was conducted using oral iron supplements. Parents completed baseline sleepdisturbance scale questionnaires for children and food-intake data and blood samples were obtained. In total, $77 \%$ of the children had restless sleep at baseline, which improved with iron supplementation. This suggests a relationship between sleep disturbance and iron deficiency in children with autism spectrum disorder.

Effective treatment of young patients with pediatric-onset, long-standing lupus nephritis with tacrolimusgiven asa single daily dose: an open-label pilot study. Tanaka H, Oki E, Tsugawa K, Nonaka K, Suzuki K, Ito E: Lupus 16(11), 896-900 (2007). Investigates the efficacy of tacrolimus, administered as a single-dose daily for maintenance therapy of young patients with pediatric-onset, long-standing systemic lupus erythematosus (SLE). A single doses of $3 \mathrm{mg} /$ day tacrolimus was administered to six patients in a 6month open-label trial. Marked improvement compared with the baseline values was observed in the European Consensus Lupus Activity M easurement index examined at 1 and 3 months. These data suggest that tacrolimus administered as a single daily dose is effective and safe for young patients with SLE.

Phase I trial and pharmacokinetic study of arsenic trioxide in children and adolescents with refractory or relapsed acute leukemia including acute promyelocytic leukemia or lymphoma. A collaborative study of The Pediatric Oncology Branch, National Cancer Institute and the Childrens Oncology Group.

Fox E, Razzouk BI, Widemann BC et al.: Blood (2007) (Epub ahead of print) .

Discusses the use of arsenic trioxide (ATO) in a Phase I trial in children to treat refractory or relapsed acute leukemia. ATO was administered intravenously over 2 h, 5 days/week for 20 doses/cycle, in two treatment groups. At lower doses, patients experience pneumonitis or neuropathic pain; at the higher dose, patients also experienced pancreatitis. Non-dose-limiting toxicities included elevated serum transaminases, nausea, vomiting, abdominal pain, constipation, electrolyte imbalance, hyperglycemia, dermatitis and headache. It is concluded that ATO is well tolerated in children at the recommended dose of $0.15 \mathrm{mg} / \mathrm{kg} /$ day.

\section{Aripiprazole decreases symptoms of mania in pediatric bipolar disorder}

A study unveiled at the Annual

$M$ eeting of the American A cademy of

Child \& Adolescent Psychiatry

(AACAP) shows that aripiprazole is effective in decreasing symptoms of mania in pediatric bipolar disorder.

The study led by Kiki Chang, Assistant Professor and Director, (Pediatric M ood D isorders Clinic, Stanford U niversity School of M edicine, CA, USA) enrolled 296 children in a 54-centre, 4-week study of patients aged 10-17 years with bipolar disease.

Patients were randomized to aripiprazole 10 or $30 \mathrm{mg}$ or placebo, and the primary end point was assessed as the mean baseline total score change in YMRS.

Results at week 4 showed that mean baseline changes in the 10 and $30 \mathrm{mg}$ study groups were significant.
There were approximately 17-point and 14-point reductions in the baseline, indicating a significant improvement in symptoms.

There are few studies in this patient population and traditional treatment consists of lithium anticonvulsants and atypical antipsychotics. A ripiprazole is currently indicated for the treatment of acute manic and mixed episodes associated with bipolar 1 disorder in adults, and for maintaining efficacy in stable patients with bipolar disorder who have had a recent manic or mixed

episode.

Source: Efficacy of aripiprazole in children (10-17) years old with mania. Presented at: Annual Meeting of the American Academy of Child \& Adolescent Psychiatry (AACAP) O ctober 23-28 Boston, MA, USA (2007) (Abstract B6)

\section{Assay for emergency diagnosis of pediatric cardiac issues}

Preliminary results have shown that B-type natriuretic peptide (BN P) testing may play an important role in the assessment of critically ill infants.

Results are based on the blood results of 33 pediatric patients admitted to Emory's pediatric cardiac intensive care unit (Atlanta, GA, U SA) with newly diagnosed congenital and acquired critical heart disease, with 60 control subjects admitted with respiratory or infectious problems.

Kevin $\mathrm{M}$ aher, a pediatric cardiologist at Emory U niversity School of M edicine (Atlanta, GA, USA), determined that the sensitivity for the assay was $100 \%$, with the specificity for diagnosing the presence of heart disease at $98 \%$. In total, $58 \%$ with positive BN P findings had congenital heart disease and 14 patients with positive tests had acquired heart disease. The diagnoses were confirmed with cardiography.

$\mathrm{H}$ eart disease symptoms in infants, which include fever, respiratory complaints, abdominal pain, fussiness and lethargy, are also consistent with the symptoms of other disorders, which makes diagnosis at speed difficult.

Access to such a test, which is now widely applied in adult cardiology, could help speed the diagnosis of seriously ill children, allowing them to reach specialist centers more quickly, where appropriate treatment can be received.

Source: American Academy of Pediatrics 2007 $\mathrm{N}$ ational $\mathrm{C}$ onference and Exhibition. $\mathrm{O}$ ctober 27-30 San Francisco, CA, U SA (2007).

\section{About the Bulletin Board}

If you have newsworthy information, please contact:

Christine Forder, Commissioning Editor,

Pediatric Health, Future Medicine Ltd., Unitec House,

2 Albert Place, London N3 1QB, UK

c.forder@futuremedicine.com

Fax: $+44(0) 2083432313$ 


\section{Sleep problems linked to children with Asperger syndrome}

\begin{abstract}
$N$ ew research published in the N ovember issue of the journal Sleep, has indicated that children with Asperger's syndrome (AS) have a higher prevalence of sleep problems. The problems are mainly related to initiating sleep, restlessness, daytimesleepinessand morning problems. This is the first study to attempt to evaluate the sleep patterns of children with AS, adjusting for sleep architecture and the cyclic alternating patterns.

Thestudy focused on eight children with AS, ten children with autism and 12 healthy control subjects. The children's sleep patternswereassessed using various material filed out by the parents. Sleep questionnaires, Pediatric $D$ aytimeSleepiness Scale, Autism Diagnostic O bservation Schedule and the Child Behavior Checklist, along with IQ tests. All children underwent an overnight polysomnogram to study the child while sleeping.
\end{abstract}

Several sleep parameters, such as time in bed, sleep-period time, number of awakenings per hour and sleep efficiency, were evaluated.

The results showed that $50 \%$ of the children with AS were reluctant to go to bed, with $75 \%$ desiring a type of stimulation such as television or a light in the bedroom. A total of $87 \%$ had difficulty initiating sleep at the start of the night, with $75 \%$ falling sleep sweating.

CAP scores were also investigated. This is a period of EEG activity during non-rapid eye movement sleep, characterized by short sequences and scored in two phases. Subjects showed a lower total CAP rate in some but not all of the sleep stages.

"T his study showed peculiar CAP modifications in children with $\mathrm{AS}$ and represented an attempt to correlate the quantification of sleep EEG oscillations with the degree of mental ability or disability", according to O liviero Bruni, from the Center for Pediatric Sleep $D$ isorders at the U niversity La Sapienza, Rome, Italy.

AS is one of several autism spectrum disorders characterized by difficulties in social interaction and by restricted and stereotyped interests and activities. AS is distinguished from the other autism spectrum disorders in having no general delay in language or cognitive development.

Current guidelines recommend that children aged between 1 and 4 years should get approximately 11-13 h sleep each night. Children aged over 5 years require 10-11 h each night, whereas adolescents require approximately $9 \mathrm{~h}$ a night. Source: Bruni O, Ferri R, Vittori E: Sleep architecture and N REM alterations in children and adolescents with Asperger syndrome. Sleep 30(11), 1577-1585 (2007).

\section{Tongue-tie treatment solves breastfeeding problems}

So called 'tongue-tie' abnormalities are thought to be responsible for breastfeeding problems in

newborn babies.

Pediatric surgeon M ervyn Griffiths (Wessex R egional Center for Pediatric Surgery, Southampton, U K) believes that a simple surgical correction in the first few months of life could result in an immediate improvement in breastfeeding.

A tongue tie is a band of tissue extending from the tongue to the floor of the mouth. Its severity varies, according to Griffiths, from abnormalities involving just the tongue tip to thick bands of tissue that secure the base of the tongue to the mouth floor.

Up to $10 \%$ of babies will have tongue tie, with approximately half of these tongue ties being severe enough to cause breastfeeding problems. The infants typically have trouble latching onto a breast during feeding and this can also cause pain for the mother.

"T he child feeds al most continually [and the] mother becomes frustrated and sore", G riffiths added.

"The child feeds a lmost continually [and the] mother becomes frustrated and sore."

Griffiths enrolled subjects for a doubleblind clinical trial, to undergo tongue-tie surgery, which some pediatric surgeons refuse to treat on the ground that it is unnecessary and they do not contribute to the problems seen in breast feeding.

In total, 60 breast-feeding mothers and babies were enrolled in a prospective double-blind, randomized, controlled trial, with a mean age of 32 days, all showing signs of known tonguetie deformities. Two groups were randomly assigned; group 1 received corrective surgery while group 2 experienced no intervention.

The babies were immediately returned to their mothers for breastfeeding after treatment or sequestration. The result indicated that $78 \%$ of women whose infants received corrective surgery said breastfeeding had improved compared with $47 \%$ of mothers whose infants were not treated.

It has been hailed as 'a simple, safe, and succesfful intervention' with very low adverse-outcome rates.

Follow-up revealed that $90 \%$ of women in the surgery group still reported improved feeding compared with $55 \%$ of mothers in the nonsurgical group.

Source: American Academy of Pediatrics $\mathrm{N}$ ational Conference and Exhibition: 0 ctober 27-30 San Francisco, CA, USA, (2007) (Abstract 39). 